\title{
Morphometric analyses of the body and the branches of the normal third interosseous muscle (suspensory ligament) in Standardbreds
}

Mohamad Khir Shikh Alsook ${ }^{\mathrm{a}, *}$, Nadine Antoine ${ }^{\mathrm{a}}$, Joëlle Piret $^{\mathrm{a}}$, Nassim Moula ${ }^{\mathrm{b}}$, Valeria Busoni $^{\mathrm{c}}$, Jean-Marie Denoix ${ }^{\mathrm{d}}$, Annick Gabriel ${ }^{\mathrm{a}}$

a Department of Morphology and Pathology, Faculty of Veterinary Medicine, University of Liege, Belgium.

b Department of Animal production, Division of Genetic, Biostatistics and Rural Economics, Faculty of Veterinary Medicine, University of Liege, Belgium.

c Department of Medical Imaging, Faculty of Veterinary Medicine, University of Liege, Belgium.

d Université Paris-Est, Ecole Nationale Vétérinaire d'Alfort, CIRALE -14430

Goustranville, France.

*Corresponding Author.

E-mail address: MVET79@HOTMAIL.COM (Mohamad Khir Shikh Alsook)

\section{Summary}

The third interosseous muscle (suspensory ligament, TIOM) is composed of connective tissue (CT) with a variable proportion of muscle (MT) and adipose tissue (AT). The aim of our study is to quantify the CT, MT and AT within the body and the branches of right thoracic and pelvic limbs TIOM in sound horses to determine whether there are differences in CT, MT and AT between age, sex, limbs and levels. Right limbs from 11 sound horses were collected. Samples from 6 levels of the TIOM were embedded in paraffin or in Tissue-Tek ${ }^{\circledR}$. Most of the paraffin sections were shredded. Using the cryosection, some artefacts appeared. Cryoprotection was carried out, which produced the best results. Hematoxylin-phloxinesaffron and Hematoxylin-eosin gave a good contrast of colours between the tissues observed allowing the use of an image analysis programme to calculate percentage of each tissue within the TIOM. The percentage of MT and AT decreased significantly $(P<0.0001)$, whereas the percentage of CT increased significantly $(P<0.0001)$ with age and when descending from the proximal to the distal level of the TIOM. The percentage of MT was significantly higher $(P<0.0001)$ in females than males, while the percentage of CT was significantly higher $(P<0.0001)$ in males than females. The percentage of AT was significantly higher 
$(P=0.0278)$ in pelvic limbs than in thoracic limbs. These results confirm the variation in tissue composition within the TIOM of sound horses. 